\title{
Eficacia y costos asociados a un tratamiento ambulatorio en mujeres con depresión severa y trauma temprano
}

\author{
VERÓNICA VITRIOL G. ${ }^{1}$, ALFREDO CANCINO A. ${ }^{2}$, RAMÓN FLORENZANO U. ${ }^{3}$, \\ SOLEDAD BALLESTEROS T. ${ }^{\text {la }}$, DANIEL SCHWARTZ P. ${ }^{\text {b }}$
}

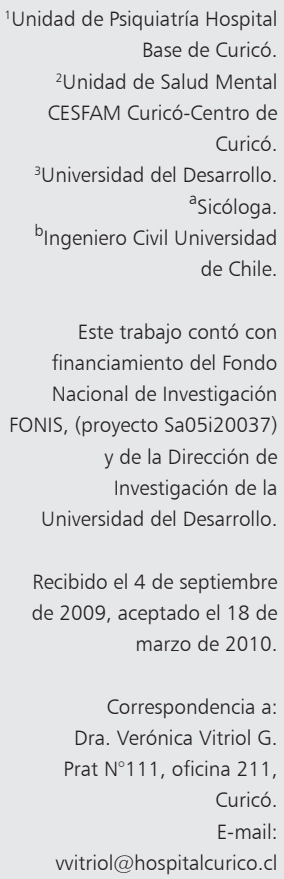

Unidad de Psiquiatría Hospital Base de Curicó.

${ }^{2}$ Unidad de Salud Mental CESFAM Curicó-Centro de Curicó. ${ }^{3}$ Universidad del Desarrollo. asicóloga. ${ }^{\mathrm{b}}$ Ingeniero Civil Universidad de Chile.

Este trabajo contó con financiamiento del Fondo Nacional de Investigación FONIS, (proyecto Sa05i20037) y de la Dirección de Investigación de la Universidad del Desarrollo.

Recibido el 4 de septiembre de 2009, aceptado el 18 de marzo de 2010

Correspondencia a: Dra. Verónica Vitriol G. Prat No111, oficina 211, Curicó. E-mail: vvitriol@hospitalcurico.cl

\section{Efficacy and associated costs of an outpatient intervention in women with severe depression and early trauma}

Background: A high proportion of women consulting for depression have a history of childhood abuse and trauma. Aim: To compare the efficacy and costs associated with a treatment that inquires directly into childhood trauma and understands present interpersonal difficulties as a compulsion to repeat the traumatic past, versus the usual treatment, in women with severe depression and childhood trauma. Material and Methods: Eighty seven women with depression and prior history of early trauma that sought help at the Mental Health Unit of the Hospital de Curicó were studied. Forty four were randomly assigned to the experimental treatment, and 43 to the usual management. Patients were evaluated using the Hamilton Depression Scale, the Outcome Questionnaire (OQ 45.2) and an expenditures sheet at baseline, three and six months. An intention to treat analysis and a simple cost-analysis were performed. Results: Hamilton and OQ 45.2 scores improved in both treatment groups, with significantly better results achieved in the experimental patients. The direct overall costs of experimental and control treatments were CLP 8,628,587 and $9,688,240$, respectively. The main contributors to costs in both arms were medications (26.5\%), followed by the number of psychiatric consultations (19.2\%) in the experimental group and by hospitalizations (25.4\%) in the control group. The costs per patient recovered in experimental and control groups were CLP 616,328 and 1,973,649, respectively. Conclusions: The proposed model resulted more effective for the treatment of this group of women.

(Rev Med Chile 2010; 138: 428-436).

Key words: Cost-benefit analysis; Depression; Stress disorders, post-traumatic.
4 n Chile, la depresión es un problema de salud pública, con prevalencias de 5,5\% para episodio depresivo durante una semana y de $9 \%$ para depresión mayor en el lapso de seis meses ${ }^{1,2}$. Se asocia a elevados costos directos e indirectos por morbimortalidad y afecta el doble a mujeres que a hombres, constituyéndose en la segunda causa de AVISA (años de vida saludable perdidos por discapacidad y muerte prematura) en el sexo femenino3. Desde el año 2006, está incluida entre las patologías con garantías explícitas en salud (GES) ${ }^{4}$. El Ministerio de Salud (MINSAL) ha estructurado una guía clínica, con indicaciones para tratar las depresiones leves y moderadas a nivel primario y las depresiones severas a nivel secundario ${ }^{5}$.

En la atención primaria, un tratamiento farmacológico escalonado al que se suman inter- 
venciones psicosociales grupales ha resultado ser más costo-efectivo que el tratamiento habitual ${ }^{6}$. En la atención secundaria, a pesar de los recursos profesionales y farmacológicos suministrados a los servicios de salud para el tratamiento de la depresión severa ${ }^{7}$, aún no existen estudios de eficacia ni análisis de costos.

Entre los factores de riesgo para el desarrollo de la depresión y el consiguiente gasto en recursos de salud, se encuentran las historias de abuso físico y sexual infantil ${ }^{8}$. Estos antecedentes se asocian a mayor complejidad y gravedad de la sintomatología depresiva, evidenciada por la presencia de comorbilidad psiquiátrica y física ${ }^{9-11}$, mala adherencia, pobre respuesta a los tratamientos farmacológicos, hospitalizaciones frecuentes, uso prolongado de días-cama y aumento de consultas en el servicio de urgencias y en el nivel primario de atención ${ }^{12-15}$.

En Chile, acorde a la evidencia internacional, se ha comprobado que los antecedentes traumáticos infantiles se pesquisan entre 60 y $80 \%$ de la población adulta consultante por trastornos depresivos a los servicios de salud ${ }^{16,17}$. Las guías clínicas nacionales vigentes para el tratamiento de la depresión aún no imparten recomendaciones específicas para el manejo de las secuelas asociadas al trauma infantil en pacientes depresivos ${ }^{5}$.

En respuesta a esta limitación, desde el año 2002, en el Hospital de Curicó se comenzó a estructurar un modelo de intervención para tratar a mujeres portadoras de depresión severa y trauma temprano ${ }^{18,19}$ cuya eficacia fue demostrada en la mejoría de los síntomas depresivos, relaciones interpersonales y rol social respecto a la intervención implementada según la Guía Clínica MINSAL ${ }^{20}$.

El objetivo de este trabajo es comparar la eficacia y los costos asociados al tratamiento propuesto, respecto de la intervención implementada conforme a las guías del MINSAL.

\section{Material y Método}

Este trabajo contó con financiamiento del Fondo Nacional de Investigación FONIS, (proyecto Sa05i20037) y de la Dirección de Investigación de la Universidad del Desarrollo, y fue autorizado por el Comité de ética del Servicio de Salud del Maule.

Diseño del estudio: Ensayo clínico aleatorio con análisis adicional de costos.

Universo y muestra: Entre los meses de abril y agosto del año 2006, todas las mujeres mayores de veinte años derivadas vía interconsulta con el diagnóstico de depresión severa $(n=154)$, fueron sucesivamente asignadas a tres psiquiatras de dilatada experiencia en el manejo de los trastornos afectivos que se desempeñan en la Unidad de Psiquiatría y Salud Mental del Hospital de Curicó.

Criterios de exclusión fueron deterioro psicoorgánico, hipoacusia, adicción actual, psicosis o antecedente de tratamiento por alguno de los psiquiatras durante el año previo al estudio.

Las pacientes con diagnóstico de depresión severa, confirmado por los especialistas según la clasificación internacional de enfermedades CIE10 $(\mathrm{n}=145)^{21}$ y sin criterios de exclusión ( $\mathrm{n}$ $=6$ ), firmaron un consentimiento informado para participar voluntariamente en este estudio; 136 pacientes accedieron a ser evaluadas por una psicóloga clínica, quien administró la escala de Hamilton de 21 ítems $^{22}$ y el screening de trauma infantil de Marshall ${ }^{23}$. Esta última escala ha sido ya utilizada en estudios previos en nuestro medio ${ }^{16,17}$.

Las pacientes con Hamilton sobre 21 puntos y que respondieron positivamente a 3 o más respuestas en la escala de Marshall o la sola respuesta positiva a contacto sexual forzado $(\mathrm{n}=$ 87), quedaron sucesivamente y aleatoriamente en dos tratamientos: el experimental $(\mathrm{n}=44)$ y el habitual $(\mathrm{n}=43)$.

El tratamiento experimental consistió en una intervención protocolizada de enfrentamiento de crisis, acorde al estado de la paciente, individual, ambulatoria, intensiva, realizada una vez a la semana por un equipo multidisciplinario durante tres meses. Ésta incorporó al tratamiento farmacológico estandarizado para la depresión severa una psicoterapia breve, cuyos objetivos fueron: abordar activamente los antecedentes traumáticos infantiles, diagnosticar la eventual comorbilidad con trastorno por estrés postraumático (TEPT) y trabajar focalizadamente en las dificultades interpersonales presentes, comprendiéndolas desde una orientación psicodinámica como una repetición del pasado traumático. Se utilizó además estrategias cognitivas, conductuales y psicoeducativas para promover el cambio de los patrones interaccionales de maltrato y agresión. El tratamiento experimental además se desarrolló en estricta coordinación entre los profesionales del equipo de Salud Mental y la inasistencia a los controles fue monitorizada mediante visitas 
y llamadas telefónicas efectuadas por la asistente social perteneciente a este equipo.

El tratamiento habitual fue desarrollado acorde a las orientaciones diagnósticas y farmacológicas vigentes difundidas en la Guía Clínica del MINSAL para la Depresión Severa, ${ }^{5,7}$, además de la psicoterapia de apoyo tradicional, que no aborda rutinariamente los traumas infantiles ni los relaciona con el motivo de consulta presente.

Ambos tratamientos fueron realizados por profesionales permanentes de la unidad de Psiquiatría del Hospital de Curicó.

Seguimiento: Las participantes fueron evaluadas al inicio, a los tres y a los seis meses de tratamiento por un equipo externo a la unidad. Una psicóloga clínica perteneciente a este equipo externo, sin estar ciega a los tratamientos, administró la escala de Hamilton. Dos alumnas de psicología, en forma ciega a los tratamientos y a los resultados del Hamilton, aplicaron el Cuestionario de Resultados de Tratamiento OQ45 de Lambert y registraron en una planilla ad-hoc los gastos incurridos en las intervenciones por persona tratada.

El OQ 45.2 de Lambert es un instrumento validado en nuestro medio. Consta de 45 ítems que abarcan el estado del paciente en tres áreas: sintomática, relaciones interpersonales y rol social. El puntaje de corte establecido para la población chilena corresponde a 73 puntos. Dicho puntaje permite discriminar una población psicológicamente funcional de una disfuncional. De acuerdo a esta prueba, se considera que existe un cambio clínicamente significativo cuando disminuye el puntaje 17 puntos respecto del inicio y éste resulta igual o menor de 73 puntos $^{24}$. La planilla de gastos fue elaborada por los autores de este trabajo, consignando los costos directos en salud mental invertidos por el servicio de salud en cada paciente durante el período de observación. Se incluyó: horas médicas, de psicólogo y de asistente social, medicamentos, hospitalizaciones, consultas de urgencia, visitas domiciliarias, contactos telefónicos y días de licencia. No fueron incluidos los gastos derivados de otra patología médica ni quirúrgica. La información se obtuvo de las fichas clínicas, del sistema de registro del protocolo propuesto y de los RUT consignados en el servicio de urgencia, internaciones y oficina de licencias médicas del hospital. Estos datos fueron valorizados según los aranceles FONASA para hospitales públicos al año2006.
Procesamiento y análisis de la información: El resultado principal para los efectos de este reporte fue el número de pacientes que puntuó para los criterios de funcionalidad según el OQ45.2. Los resultados de las diversas escalas fueron procesados estadísticamente mediante el paquete computacional SPSS 1.2, considerándose el análisis por intención de tratar. La significación estadística utilizada fue de $\mathrm{p}<0,05$. Se realizó un análisis simple de costos que comparó la suma del total de gastos invertidos en las pacientes de cada grupo durante el período de observación, dividida por el número de pacientes que a los seis meses mejoraron significativamente sus puntajes en la escala global del OQ45.2.

\section{Resultados}

La adherencia a los tratamientos entre ambos grupos fue similar. El 91\% $(\mathrm{n}=79)$ de la muestra fue evaluada a los tres meses ( 39 del experimental, 40 del control) y $81 \%(\mathrm{n}=71)$ a los seis meses (36 del experimental, 35 del control). No hubo diferencias significativas entre los parámetros iniciales de las pacientes de ambos grupos que abandonaron tratamiento.

La Tabla 1 muestra que en las observaciones preliminares no se encontró diferencias estadísticas entre ambos grupos, ni en sus características socio-demográficas, ni en los promedios de los test, ni en los antecedentes traumáticos infantiles.

En cuanto a resultados de eficacia, la Tabla 2 muestra la evolución de los promedios del Hamilton y el OQ45. Se observan diferencias significativas entre ambos grupos al final de la intervención a favor del grupo experimental en el Hamilton $(t=2,69 ; p<0,001)$ y en el OQ45 $(t=$ $1,98 ; \mathrm{p}<0,05)$. Estas diferencias a favor del grupo experimental se mantienen al final del período de observación: en el Hamilton $(t=2,69 ; \mathrm{p}<0,01)$ y en el OQ45 global $(t=2,16 ; p<0,05)$. Al final del período de observación, 14 pacientes del grupo experimental y 5 del grupo control presentaron puntajes con criterios de funcionalidad según el OQ45 (38,9\% versus $14,3 \%$, respectivamente, chi cuadrado $\mathrm{p}<0,05)$.

Los resultados del estudio adicional de costos (Figura 1) muestran que el costo directo total del tratamiento experimental fue de CLP 8.628.587 y el del grupo control CLP 9.688.240. El mayor costo 
Tabla 1. Características basales de 87 mujeres con depresión severa y trauma temprano randomizadas a dos tratamientos (Curicó Chile, 2006)

\begin{tabular}{|c|c|c|c|c|c|}
\hline & \multicolumn{2}{|c|}{ Experimental } & \multicolumn{2}{|c|}{ Control } & $\mathbf{p}$ \\
\hline \multirow[t]{2}{*}{ Edad promedio } & \multicolumn{2}{|c|}{$37 \pm 10,75$} & \multicolumn{2}{|c|}{$41 \pm 11,8$} & 0,73 \\
\hline & $\mathbf{n}$ & $\%$ & $\mathbf{n}$ & $\%$ & \\
\hline \multicolumn{6}{|l|}{ Escolaridad } \\
\hline Básica & 23 & 52 & 22 & 51 & \\
\hline Media & 18 & 41 & 19 & 44 & \\
\hline Superior & 3 & 7 & 2 & 5 & 0,7 \\
\hline \multicolumn{6}{|l|}{ Estado Civil } \\
\hline Soltero & 3 & 7 & 7 & 16 & \\
\hline Separado & 7 & 16 & 8 & 18 & \\
\hline Casado & 19 & 43 & 19 & 44 & \\
\hline Viudo & 3 & 7 & 1 & 1 & \\
\hline Conviviente & 12 & 27 & 8 & 21 & 0,8 \\
\hline \multicolumn{6}{|l|}{ Ocupación } \\
\hline Dueña de casa & 21 & 48 & 24 & 56 & \\
\hline Empleada & 20 & 46 & 15 & 35 & \\
\hline Cesante & 2 & 5 & 3 & & \\
\hline Estudiante & 1 & 2 & 1 & 2 & 0,5 \\
\hline \multicolumn{6}{|l|}{ Evaluaciones iniciales } \\
\hline Hamilton $^{(1)}$ & \multicolumn{2}{|c|}{$34,8 \pm 6,3$} & \multicolumn{2}{|c|}{$34,6 \pm 6,2$} & 0,8 \\
\hline OQ45 Total(2) & \multicolumn{2}{|c|}{$112,6 \pm 20,4$} & \multicolumn{2}{|c|}{$117,5 \pm 19,7$} & 0,2 \\
\hline Marshall(3) Prom. Resp. (+) & \multicolumn{2}{|c|}{$4,2 \pm 1,3$} & \multicolumn{2}{|c|}{$4,1 \pm 1,3$} & 0,1 \\
\hline
\end{tabular}

(1)La escala de Hamilton varía entre 0 a 63 puntos: A mayor puntaje, mayor gravedad de la depresión. (2)La escala 0Q45 global varía en sus puntajes entre 0 a 180 puntos: A mayor puntaje, peor funcionamiento. ${ }^{(3)}$ La escala de Marshall indaga sobre 7 eventos traumáticos ocurridos antes de los 16 años (separación traumática del cuidador, abuso de alcohol o drogas en la familia, castigo físico, secuela de castigo físico, ser testigo de violencia intrafamiliar, contacto sexual con familiar y no familiar). No se encontró diferencias significativas en ninguno de los ítems estudiados.

en ambos tratamientos estuvo dado por el uso de los medicamentos $(26,5 \%)$. En el grupo experimental es seguido por el número de consultas psiquiátricas $(19,2 \%)$, pago de días de licencia $(8,2 \%)$ $y$ hospitalizaciones $(8,2 \%)$. En el grupo control el mayor costo en medicamentos es seguido por las hospitalizaciones $(25,4 \%)$, pago de días de licencia $(11,9 \%)$ y visitas a urgencia $(7,3 \%)$.

La Tabla 3 muestra el promedio de los costos por persona de los distintos insumos de cada grupo. Se observan diferencias estadísticamente significativas entre los grupos en el promedio de los costos por hospitalización y consultas psiquiátricas. Durante el período de observación se hospitalizó a 7 pacientes del grupo experimental y a 6 del grupo control con un promedio de días cama en el grupo control de $21,3 \pm 8$ días y $6 \pm 5$ días en el experimental $(\mathrm{p}<0,001)$. Hubo también diferencias significativas en el número de consultas psiquiátricas: el promedio de consultas psiquiátricas del grupo control fue de $2,5 \pm 2$ consultas y del experimental $7 \pm 3,7$ consultas $(\mathrm{p}<0,01)$.

La Tabla 4 describe el análisis adicional de costos realizado en el presente estudio. El costo de tratamiento para recuperar a un paciente según los indicadores del OQ45 en el grupo experimental fue de CLP 616.328 y en el grupo control de CLP 1.973.649. La diferencia entre los costos entre 
Tabla 2. Cambios en el tiempo de los promedios del Hamilton y OQ45 de 87 mujeres con depresión severa y trauma temprano asignadas a dos tratamientos (Curicó Chile, 2006)

\begin{tabular}{|lcccccc|}
\hline & \multicolumn{2}{c}{ Inicio } & \multicolumn{2}{c}{ Tres meses } & \multicolumn{2}{c|}{ Seis meses } \\
& Promedio & (DE) & Promedio & (DE) & Promedio & (DE) \\
Hamilton(1) & & & & & & \\
Grupo experimental & 34,09 & 6,2 & 22,1 & 8,8 & 19,4 & 8,8 \\
Grupo control & 34,42 & 6,7 & 27,5 & 9,7 & 25,0 & 11,1 \\
OQ45 Total(2) & & & & & & \\
Grupo experimental & 112,73 & 19,7 & 92,7 & 28,3 & 85,8 & 37,6 \\
Grupo control & 114,72 & 22,5 & 105,4 & 31,0 & 102 & 31,7 \\
\hline
\end{tabular}

${ }^{(1)}$ Ambos tratamientos mejoraron en la escala de Hamilton: El grupo experimental (Pillai's trace $=0,49, \mathrm{~F}=38,85, \mathrm{gl}=2 \mathrm{y}$ $42, p<0,01$ ) y el control (Pillai's trace $=0,51, F=21,39, d f=2$ y $41, p<0,01$ ). Hubo diferencias significativas entre ambos grupos a favor del experimental a los tres meses $(p<0,001)$ y a los seis meses $(p<0,01)$. ${ }^{(2)}$ Ambos tratamientos mejoraron significativamente en el OQ45 global: El experimental (Pillai's trace $=0,42, F=18,60, d f=2$ y $42, p<0,001$ ) y el control (Pillai's trace $=0,21, F=5,70, d f=2$ y $41, p<0,01$ ). Hubo diferencias significativas a favor del grupo experimental a los tres y seis meses $(p<0,05)$.

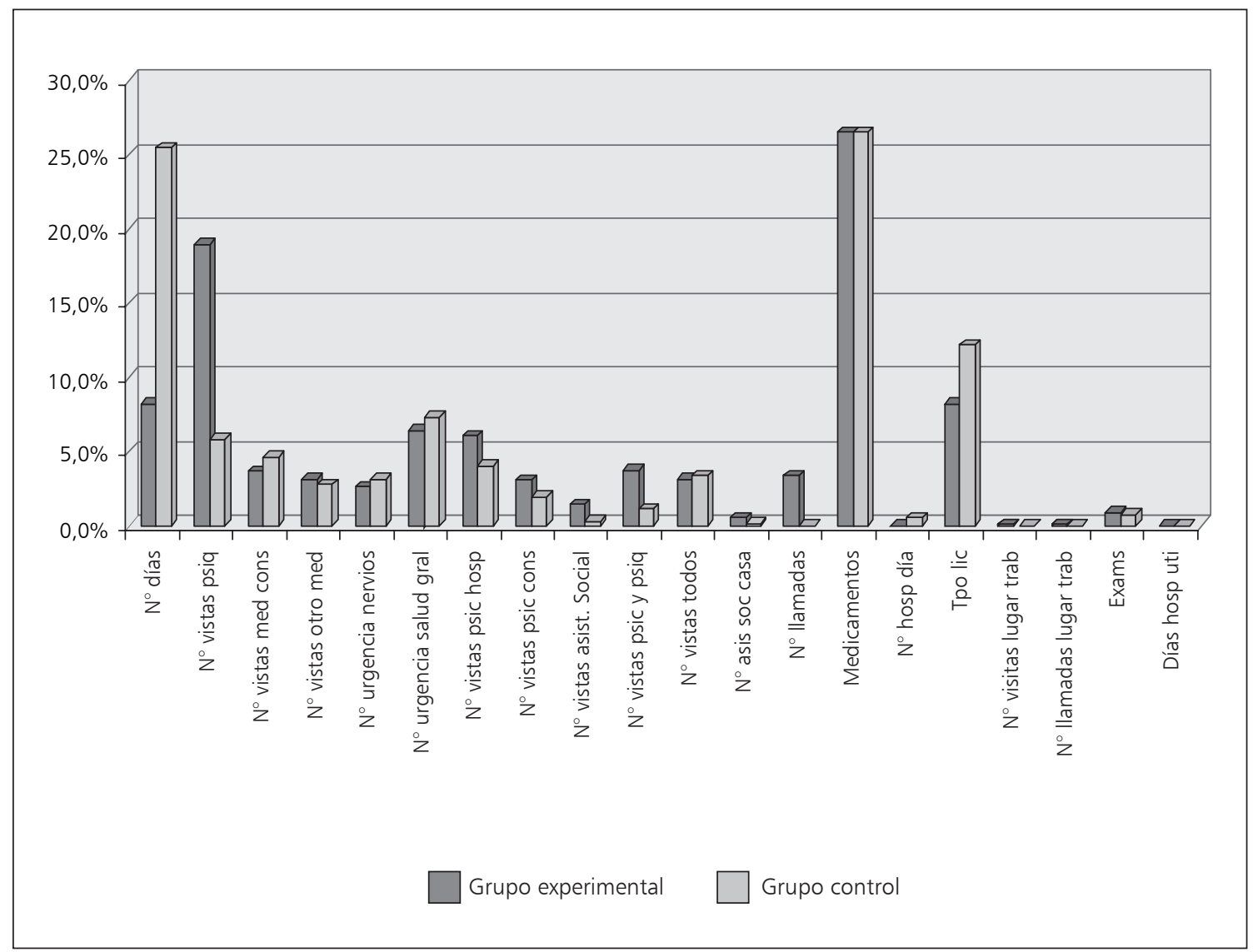

Figura 1. Proporción de costos de dos tratamientos en mujeres con depresión severa y trauma temprano (Curicó 2006). 
Tabla 3. Promedio de costos en CH\$ utilizados por 87 mujeres consultantes por depresión severa

\begin{tabular}{|c|c|c|c|c|}
\hline \multirow[t]{2}{*}{ Tipo de insumos(1) } & \multicolumn{2}{|c|}{ Grupo experimental(2) } & \multicolumn{2}{|c|}{ Grupo control ${ }^{(2)}$} \\
\hline & CLP (m) & DE & CLP (m) & DE \\
\hline Días de hospitalización* & 16,15 & 13,4 & 58,37 & 21,9 \\
\hline Consultas psiquiátricas* & 37,2 & 19,5 & 13,38 & 10,5 \\
\hline Consultas médico APS & 7,2 & 10,8 & 10,6 & 11,2 \\
\hline Cons. smb. otro médico hospital & 6,07 & 10,2 & 6,3 & 8,7 \\
\hline Cons. urgencia por sínt. emocionales & 5,11 & 12,0 & 7,2 & 10,13 \\
\hline Cons. urgencia por sínt. generales & 12,5 & 22,3 & 16,8 & 29,2 \\
\hline Cons. amb. psicólogo hospital & 11,7 & 7,2 & 9,2 & 10,0 \\
\hline Cons. psicólogo APS & 6,0 & 16,5 & 4,5 & 9,3 \\
\hline Cons. amb. a. social hospital & 2,8 & 3,09 & 0,6 & 1,44 \\
\hline Entrev. por eq. multiprofesional & 7,3 & 4,4 & 2,5 & 5,1 \\
\hline Intervención familiar x AS & 6,12 & 8,66 & 7,6 & 16,37 \\
\hline Llamadas telefónicas A.S.** & 6,62 & 4,98 & 0,027 & 0,23 \\
\hline Medicamentos $^{(3)}$ & 51,99 & 42,11 & 60,85 & 78,3 \\
\hline Tiempo promedio licencia & 16,13 & 13,87 & 27,94 & 13,97 \\
\hline
\end{tabular}

(1)Valores de los insumos FONASA 2006: día cama medicina 40.510, consulta psiquiatría 8.940, consulta médico AP 7.090, consulta médico urgencia 16.550, consulta psicólogo hospital 4.990, consulta psicólogo CRS 7.090, consulta asistente social 8.090, visita domicilio AS 6.410, entrevista en equipo 15.640. (2) Todos los costos se dividieron por el total de la población ( $\mathrm{n}=$ 44 exp. y $n=43$ control). ${ }^{(3)}$ Costo en CLP por comprimido de medicamentos (Fluoxetina 19, Clonazepam 87, Carb. de litio 19, Lamotrigina 857, Ácido Valproico, 44, Carbamazepina 238, Haloperidol 109, Clorpromazina 15, Amitriptilina 6 , Alprazolam 7, Risperidona 81). ${ }^{*} p<0,01 .{ }^{* *}$ Llamadas telefónicas: valorizadas en función del tiempo/profesional invertido por A.S. en efectuarlas.

Tabla 4. Análisis de costos a los seis meses de observación de los dos tratamientos en mujeres con depresión severa y trauma temprano (Curicó, 2006)

\begin{tabular}{|c|c|c|c|c|}
\hline Tratamientos & & Experimental & Control & Diferencias \\
\hline Costo total & $(\mathrm{CLP})$ & 8.628 .587 & 9.868 .244 & 1.239 .657 \\
\hline OQ45 funcionales & (n) & 14 & 5 & 9 \\
\hline Costo por paciente & $(C L P)$ & 616.328 & 1.973 .244 & $\begin{array}{c}137.739 \\
\text { (beneficio incremental) }\end{array}$ \\
\hline
\end{tabular}

ambos grupos, dividido por la diferencia entre los pacientes que cumplían el criterio de recuperación según el OQ45 permite obtener un beneficio incremental de CLP 137.739.

\section{Discusión}

Este estudio fue realizado en una población con indicadores de alta gravedad de la sintomatología depresiva, suicidalidad y disfuncionalidad ${ }^{18}$. En dicha población el nuevo modelo implementado no sólo demostró ser más eficaz en la mejoría de los síntomas depresivos y generales que el tratamiento habitual, sino que además resultó ser más económico. No se puede determinar si estos resultados son consecuencia de la orientación clínica utilizada o de la gestión ambulatoria de los recursos en salud implementada. Sin embargo, ambos aspectos, en este tratamiento estuvieron interconectados. Este modelo gestionó los recur- 
sos suministrados para tratar la depresión severa administrándolos en consideración del tipo de vínculo que los sujetos traumatizados requieren establecer con los profesionales del equipo de salud para que puedan trabajar en sus dificultades interpersonales ${ }^{25}$.

Dichos resultados locales son concordantes con estudios previos que demuestran cómo los tratamientos ambulatorios y estructurados, que incluyen recursos tan económicos como los contactos vía telefónica, son de mayor costoefectividad en el tratamiento de pacientes con depresión ${ }^{26}$. Coinciden, además, con la evidencia empírica que demuestra que en pacientes psicotraumatizados los tratamientos intrahospitalarios breves o ambulatorios son más costo-efectivos que las internaciones prolongadas ${ }^{27}$. De hecho, existen reportes de recaídas sintomáticas relevantes luego del alta intrahospitalaria ${ }^{28}$. Una explicación para este fenómeno podría sustentarse en el hecho que, durante la internación, se aísla al paciente de las dificultades habituales presentes en su contexto psicosocial, las cuales deben ser nuevamente enfrentadas al retornar a su medio. Esta situación no sucedió con el nuevo modelo implementado, lo que constituye una de sus fortalezas.

Otra de las fortalezas de esta investigación estriba en su validez externa ya que ha sido diseñada y realizada por los propios profesionales de un equipo de salud mental y en las condiciones habituales de trabajo de un hospital público de Chile ${ }^{29}$.

El mencionado modelo de atención amerita involucrar a los profesionales de la atención primaria, para llegar a constituirse en una intervención coordinada y continua entre el nivel especializado y el nivel primario de atención, puerta de entrada inmediata de la demanda asistencial. Dichos profesionales debieran contar con las competencias clínicas necesarias para realizar: la evaluación integral de este tipo de usuario (considerando la indagación de antecedentes traumáticos tempranos, actuales, o ambos), la formulación diagnóstica inicial de la depresión y del eventual TEPT comórbido, y el tratamiento de mantención a las pacientes ya estabilizadas.

Los resultados del estudio de costos de este trabajo -obtenidos desde un análisis simple utilizando un instrumento validado en la población chilena- requieren ser complementados con un estudio de costo-efectividad ${ }^{30,31}$ que incluya, entre otros factores, la valorización del tiempo- profesional que demanda el diseño y la capacitación sobre este modelo (en este estudio, este ítem fue asimilado al quehacer habitual de estos facultativos) y cuyo denominador sea "días libres de enfermedad" usualmente empleado en diseños de costo-efectividad en pacientes con patología mental $^{32}$. Sin embargo, los autores consideran que las limitaciones mencionadas no representan un factor que distorsione las conclusiones obtenidas a partir de esta investigación, toda vez que pudo establecerse confiablemente que una comprensión clínica de las secuelas de los traumas tempranos en sujetos con depresión no sólo fue más eficaz, sino que implicó un tipo de gestión de los recursos que permitió un ahorro de costos a nivel local y que podría tener aún un mayor impacto al implementar este modelo involucrando el nivel primario de la red asistencial ${ }^{33}$. En tal sentido, estas nuevas perspectivas están siendo consideradas en la revisión de la guía clínica para el tratamiento de la depresión que actualmente está realizando el MINSAL. En resumen, se puede concluir que el modelo propuesto, que indaga activamente en los traumas tempranos y comprende las dificultades interpersonales presentes como una repetición del pasado traumático en mujeres con depresión severa y trauma temprano (quienes constituyen una fuente de alta demanda en los servicios de salud), resultó ser más eficaz y promete ser más costo-efectivo. Dada la gravedad y la tendencia a la cronicidad de estas pacientes ${ }^{34}$, se requiere continuar validando estrategias de tratamiento a largo plazo que involucren a toda la red de salud, e incorporar al modelo ya elaborado estudios de costo-efectividad, que evalúen los gastos directos e indirectos para la salud pública (incluidos los de aquellos pacientes que no adhirieron al tratamiento).

\section{Referencias}

1. Vicente B, Rioseco P, Saldivia S, Kohn R, Torres S. Estudio chileno de prevalencia de patología psiquiátrica (DSM-III-R/CIDI) (ECPP). Rev Med Chile, 2002; 130: 527-36.

2. Araya R, Rojas G, Fritsch R, Acuña J, Lewis G. Common mental disorders in Santiago, Chile: prevalence and sociodemographic correlates. Br J Psychiatry 2001; 178: 228-33.

3. Informe Final Estudio de carga de enfermedad y carga 
atribuible. Gobierno de Chile, Ministerio de Salud, 2007.

4. Decreto de aprobación de Problemas de Salud y Garantías Explícitas en Salud de la Ley 1996. Gobierno de Chile, Ministerio de Hacienda- Salud, 2005.

5. Ministerio de Salud Chile. Guía Clínica para el Tratamiento de Personas con Depresión. Unidad Salud Mental, 2006.

6. Araya R, Rojas G, Fritsch RM, Gaete J, Rojas M, Simon $\mathrm{G}$, et al. Treating depression in primary care in low-income women in Santiago, Chile: A randomized controlled trial. Lancet 2003; 361: 995-1000.

7. Programa Nacional de Detección, Diagnóstico y Tratamiento de los Trastornos Depresivos en el Nivel primario de Atención. Algoritmos y Flujogramas para el Tratamiento de la Depresión Severa, Ministerio de Salud [Avances] Santiago Chile, Unidad de Salud Mental, 2003-2004.

8. Kendler K, Kuhn J, Prescott C. Childhood sexual abuse, stressful life events and risk for mayor depression in women. Psychol Med 2004; 36: 1475-82.

9. Van Der Kolk B, Roth S, Pelcovitz D, Sunday S. Disorders of extreme stress: the empirical foundation of a complex adaptation to trauma. J Trauma Stress 2005; 18: 389-99.

10. Oquendo M, Brent D, Birmaher B, Greenhill L, Kolko D, Stanley D. Posttraumatic stress disorder comorbid with major depression: factors mediating association with suicidal behavior. Am J Psychiatry 2005; 162: 560-6.

11. Sledjeski E, Speisman B. Does number of life traumas explain the relationships between PTSD and chronic medical conditions? Answers from the National Comorbidity Survey-Replication (NCS-R). J Behav Med 2008; 31: 341-9.

12. Trivedi M, Rush J, Wisnekie S, Nierenberg A, Warden D, Ritz L et al. Evaluations of citalopram for depressions using measurement based on STAR-D: implications for clinical practice. Am J Psychiatry 2006; 163: 28-40.

13. Holtzheimer P, Russo J, Zatzick D, Bundy C, RoyByrne P. The impact of comorbid posttraumatic stress disorder on short-term clinical outcome in hospitalized patients with depression. Am J Psychiatry 2005; 162: 970-6.

14. Chan D, Cheadle AD, Reiber G, Unutzer J, Chaney EF. Health care utilization and its costs for depressed with and without comorbid PTSD symptoms. Psychiatr Serv 2009; 16: 1612-7.

15. Walter E, Katon W, Russo J, Ciechanowski P, Newman E, Wagner A. Health care costs associated with posttraumatic stress disorder symptoms in women. Arch Gen Psychiatry 2003; 60: 269-374.
16. Weil K, Florenzano R, Vitriol V, Cruz C, Carvajal C, Fullerton C, et al. Trauma infanto juvenil y psicopatología adulta: Un estudio empírico. Rev Med Chile 2004; 132: 1499-504.

17. Vitriol V. Relación entre psicopatología adulta y antecedentes de trauma infantil. Rev Chil Neuro-Psiquiat 2005; 43: 83-7.

18. Ballesteros S, Vitriol V, Florenzano R, Calderón A, Vacarezza A. Mujeres con depresión severa: Relación entre trauma infantil y gravedad de síntomas clínicos. Rev Neuro-Psiquiat 2007; 45: 288-95.

19. Vitriol V, Gomberoff M, Basualto M, Ballesteros S. Relación entre trastorno por estrés postraumático de inicio tardío, abuso sexual infantil y revictimización sexual: Caso Clínico. Rev Med Chile 2006; 10: 1303-5.

20. Vitriol V, Ballesteros S, Florenzano R, Weil K, Benadof D. Evaluation of an outpatient intervention for women with severe depression and a history of childhood trauma. Psychatr Serv 2009; 60: 636-42.

21. Clasificación Internacional de Enfermedades (OMS) Descripciones Clínicas y pautas para el Diagnóstico. Madrid: Ediciones Meditor, 1992.

22. Hamilton M. A rating scale for depression. J Neurol Neurosurg Psychiatry 1960; 23: 56-62.

23. Cuneo C, González I, Jara M, Palomares L, Rammasy C, Cruz C, et al. Validación externa de la Escala de Trauma de Marshall. En: Florenzano R, Weil P, Carvajal C, Cruz C: Trauma Infanto-Juvenil y Psicopatología adulta. Santiago: Editorial Corporación de Promoción Universitaria, 2005.

24. Von Bergen A, De la Parra G. OQ-45.2, Cuestionario para evaluación de resultados y evolución de psicoterapia: Adaptación, validación e indicaciones para su aplicación e interpretación. Rev Ter Psicológica 2002; 20: 161-75.

25. Cloitre M, Cohen L, Koenen K. Treating survivors of childhood abuse psychotherapy for the interrupted life. New York-London, Guilford Press, 2006.

26. Fritsch R, Araya R, Solís J, Montt E, Pilowsky D, Rojas $\mathrm{G}$. [A randomized trial of pharmacotherapy with telephone monitoring to improve treatment of depression in primary care in Santiago, Chile]. Rev Med Chile 2007; 135: 587-95.

27. Fontana A, Rosencheck R. Effectiveness and cost of the inpatient treatment of posttraumatic stress disorder: Comparison of three model of treatment. Am J Psychiatry 1997; 154: 758-65.

28. Stalker C, Palmer S, Wright D, Geboyts R. Specialized inpatient trauma treatment for adults abused as children: a follow up study. Am J Psychiatry 2005; 162: 552-9.

29. Rosemberg S, Mueser K, Friedman M, Gorman P, Drake 
Eficacia y costos asociados a tratamiento ambulatorio de depresión y trauma temprano - V. Vitriol G. et al

R, Vivader R, et al. Developing effective treatments for posttraumatic stress disorders among people with severe mental illness. Psychiat Serv 2001; 52: 1453-61.

30. Drummond M, O'Brien B, Stoddard G, Torrance G. Métodos para la evaluación económica de los programas de asistencia sanitaria. Madrid. Ediciones Diez Santos, 2001.

31. Hoch J, Smith M. A guide to economic evaluation. Methods for cost-effectiveness analysis of person - level data. J Trauma Stress 2006; 19: 787-97.

32. Araya R, Flynn T, Rojas G, Fritsch R, Simon G. Cost- effectiveness of a primary care treatment program for depression in low-income women in Santiago, Chile. Am J Psychiatry 2006; 163: 1379-87.

33. Cancino A, Asenjo M, Jofré X. Estudio de antecedentes traumáticos infantiles en personas ingresadas al programa de depresión en el CES Curicó- Centro durante el año 2006. Rev FACE-ESGS 3 2007; 7: 1-16.

34. Wiersma J, Hovens J, Von Oppen P, Gitay E, Van Schaik D, Beekman A. The importance of childhood trauma and childhood life events for chronicity of depression in adults. J Clin Psychiatry 2009; 70: 983-9. 